\title{
Circuit
}

Musiques contemporaines

\section{Le quatuor à cordes est une forme tabou, un fossile et il n’appartient plus à une tradition vivante}

\section{John Rea}

Volume 11, numéro 2, 2000

Le quatuor à cordes selon Schafer

URI : https://id.erudit.org/iderudit/004695ar

DOI : https://doi.org/10.7202/004695ar

Aller au sommaire du numéro

Éditeur(s)

Les Presses de l'Université de Montréal

ISSN

1183-1693 (imprimé)

1488-9692 (numérique)

Découvrir la revue

Citer cet article

Rea, J. (2000). Le quatuor à cordes est une forme tabou, un fossile et il n’appartient plus à une tradition vivante. Circuit, 11(2), 63-66.

https://doi.org/10.7202/004695ar d'utilisation que vous pouvez consulter en ligne. 


\title{
Le quatuor à cordes est une forme tabou, un fossile et il n'appartient plus à une tradition vivante
}

\author{
John Rea
}

Pour faire suite à la citation de Pierre Boulez et aux questions posées par Jean Portugais, selon moi, deux perspectives s'offrent à nous et ont, décidément, une connotation religieuse (comme en témoigne, par exemple, l'emploi des termes "tabou », "écriture épurée », "besoin d'ascétisme ", "enjeu sacré » ou "épreuve initiatique ». On peut en venir rapidement à une conclusion: ce genre du quatuor à cordes est l'objet d'un culte.

\| est comme un "objet» appartenant au catholicisme romain, c'est-à-dire un ostensoir, mais un ostensoir sonore, semblable à la pièce d'orfèvrerie en forme de soleil rayonnant destinée à contenir l'hostie consacrée et à l'exposer à l'adoration des fidèles. L'ostensoir en or brillant est montré durant la cérémonie dite de la Bénédiction. Dans le cadre du catholicisme romain, cette action liturgique accompagne la notion doctrinaire de la présence réelle du Christ dans I'hostie, le dogme de la transsubstantiation.

Donc, il existe selon moi un "culte» musical particulier, dont l'un des objets d'adoration est le quatuor à cordes; appelons cela "concertisme» de musique de chambre. Suivant une logique métaphorique inévitable, nous sommes portés à compléter le tableau liturgique : I'auditoire dans la "chambre d'écoute " est la congrégation assise à l'église, l'assemblée des fidèles; les musiciens sur scène, les membres du quatuor à cordes, sont le prêtre ou la prêtresse et ses cocélébrants tenant l'ostensoir en l'air Ulean Portugais a demandé si la "musique pure » était plus "élevée»); et puis, l'hostie - le corps, le sang, l'âme et la divinité du... compositeur/Christ, le fils de Dieu! Je dis bien le fils, et non pas, la fille de Dieu!

La présence réelle du compositeur/Christ dans I'hostie sonore, ce qu'on pourrait nommer le dogme de la transsubstantiation musicale, est à la base, donc, de la déférence accordée par des fidèles aux créateurs, déjà dans l'au-delà éternel. 
Surtout quant il s'agit des seize lou dix-sept, en comptant la Grosse Fuge op. 133) quatuors à cordes de Beethoven (en particulier les six derniers dans lesquels le compositeur/Christ fait face à la mort !), ou les quinze quatuors de Chostakovitch, cette figure pathétique "crucifiée » par sa société insensible. II me semble que ce dogme nous mène toujours à une condition fatale.

D'ailleurs, le philosophe Theodor Adorno, lorsqu'il faisait une référence au dernier style de Beethoven, constatait que cette manière d'écrire était la manifestation des premiers symptômes d'une condition fatale, condition déjà en cours, ou sur le point de l'être pour la musique moderne contemporaine (Adorno, in Subotnick, 1976).

Et qu'est-ce que tout cela aurait à voir avec l'objet de notre discussion? Laissez-moi vous donner quelques points de repères; il y en aura neuf:

(1) Depuis une bonne trentaine d'années, les compositeurs tentent de "recomposer » le quatuor à cordes; ce sont de faux prophètes ou messies. Ue vous rappelle que le mot «messie» vient de l'araméen [meschikhâ, qui veut dire "oint du Seigneur »], à travers l'hébreu [mâschiakh] puis, traduit par les Grecs, en khristos.)

(2) Le culte appelé "concertisme de musique de chambre » n'existe plus, parce que la chambre est devenue un stade, sinon, le Colisée.

(3) Une œuvre récente pour "quatre instruments à cordes amplifiés», écrite spécifiquement pour le Kronos Quartet par le compositeur néerlandais Lovis Andriessen, porte un titre de mauvais augure, sinon de malheureux pressentiment - Facing Death (faire face à la mort), un titre éloquent.

(4) Comme tous les autres faux prophètes, un compositeur de quatuor à cordes postmoderne - car, c'est ce qu'il est - tente une action hérétique : il essaie de déplacer son corps, son sang, son âme et sa divinité loin de l'ostensoir sonore vers le diffuseur, le haut-parleur, l'outil essentiel au prédicateur criard. L'hostie ne se retrouve plus au sein du quatuor et, donc, l'autre cérémonie liturgique appelée la Communion ne peut même plus avoir lieu: le communiant ne reçoit pas le contenu de la composition. II n'y en a pas! Je dirai que Facing Death veut dire aussi... faire face à son vide intérieur en tant qu'artiste.

(5) Parce que la profession de foi judéo-chrétienne a été abandonnée par tous au profit d'une nouvelle religion, plus cosmopolite - une "géo-religion" quoi ? - , plus rassurante et pleine de vivants et véritables saints esprits qui marchent sur la terre, que le géo-économiste américain Edward Luttwak appelle le "capitalisme-turbo", donc parce que la profession de foi judéo-chrétienne a été abandonnée au profit du capitalisme-turbo, le contenu spirituel de ce genre instrumental qu'est le quatuor à cordes a été évacué. Le professeur Luttwak précise que le capitalisme-turbo est né à la fin des années soixante-dix. C'est le moment, par hasard ou par volonté, où le Kronos Quartet commençait à vendre ces «produits postmodernes»! 
(6) Grâce au marché international du disque - le disque, un objet en forme d'hostie - il existe un nouveau sacrement, une nouvelle Bénédiction urbi et orbi, que célèbrent les compagnies dans l'industrie culturelle transnationale. Ils standardisent tout l'art musical, le convertissent en marchandise, puis, ils vous trompent en vous disant que cet art est «original» (ce que Adorno appelle la "pseudoindividualité » [1992]). Par la suite, et par une publicité "théologiquement correcte », ils vous vendent l'hostie en plastique, prêt-à-porter des diffuseurs, les hautparleurs, chez vous. Par conséquent, et suivant encore l'argument de Adorno, l'audition de cette musique est "régressive», c'està-dire qu'elle vous oblige à redevenir tel un enfant devant l'œuvre. Cette machine culturelle infernale vous transforme : elle met votre conscience et votre esprit critique en sourdine.

(7) Leur conscience étant mise en sourdine, les jeunes compositeurs (ici je parle de l'homme) ne prétendent plus être "créateurs"; ne savent pas jouer le rôle du Christ; ne sont pas attirés par la notion de Communion. Néanmoins, ils cherchent à tout prix une Bénédiction urbi et orbi en écrivant des musiques dictées par les géo-capitalistes-turbo. Ils ne composent plus de quatuors à cordes authentiques.

Les jeunes compositrices, par contre, avec leurs consciences piquées par une autre religion, le "féminisme-macho", se regardent dans le miroir, voient la femme devenue homme, mais elles se disent: "Nous ne voulons plus être maltraitées, ni crucifiées. " Donc, ne prétendant jamais être "créateurs", ne voulant pas jover le rôle du Christ, n'étant pas attirées par la notion de Communion parce que la femme n'est pas un objet - n'est pas un acquêt susceptible d'appropriation ou d'ingestion - elles cherchent, néanmoins, une Bénédiction urbi et orbi en écrivant des musiques dictées par les géo-capitalistes-turbo. Elles ne peuvent pas composer de quatuors à cordes authentiques.

(8) La montée du capitalisme ancien, il y a 250 ans, coïncide avec l'ascension du genre ancien du quatuor à cordes, et ce n'est pas par hasard. Le dieu du Temps, Kronos, a-t-il manipulé ainsi les deux destins? Je pense que oui!

Pour la haute bourgeoisie de cette époque, la "chambre d'écoute», le chezsoi, avait une fonction exclusive, celle d'être un espace clos où l'on pouvait assister à, voire se baigner dans, une sorte de liturgie musicale qui faisait contraste avec le monde extérieur et avec le mouvement psycho-économique alors naissant qui concordait avec l'arrivée du libéralisme international. Aujourd'hui, grâce à la télévision, au lecteur laser et, de plus en plus, à l'Internet, l'extérieur se retrouve à l'intérieur: le marché est le chez-soi! La chambre d'écoute est devenue une salle d'un entrepôt ou une kermesse bruyante.

(9, et dernier point) Le quatuor à cordes fait face à la mort, is facing death. II se peut que le genre soit déjà mort. Nous nous comportons ici, autour de la table, comme des médecins qui examinent un cadavre. Bientôt, on conclura avec la radiographie que même l'os est atteint. Le fait même d'imaginer la nécessité d'en parler encore indique, selon moi, une prise de conscience qui nous prend 
à la gorge: "Oui, c'est vrai, dit cette petite voix intérieure râpeuse, nous ne nous sommes pas rendu compte que l'état des choses était si grave, que sa condition... était fatale».

\section{Bibliographie}

ADORNO, T. W. (1992) [1963], "Commodity Music Analyzed", Quasi una Fantasia, trad. en anglais par R. Livingstone, Londres, Verso Publications, 336 p.

ANDRIESSEN, L. (1990), Facing Death, for four amplified string instruments, Londres, Boosey \& Hawkes. Partition.

LUTTWALK, E. (1999), Turbo Capitalism, New York, Harper Collins, 290 p.

SUBOTNIK, R. R. (1976), "Adorno's Diagnosis of Beethoven's Late Sytle: Early Symptoms of a Fatal Condition ", Journal of the American Musicological Society, vol. 29, n² 2, été, p. 242-275. 